\title{
A Hybrid Electrooptic Microring Resonator-Based $1 \times 4 \times 1$ ROADM for Wafer Scale Optical Interconnects
}

\author{
Jocelyn Takayesu, Michael Hochberg, Tom Baehr-Jones, Eric Chan, Guangxi Wang, Philip Sullivan, Yi Liao,
} Josh Davies, Larry Dalton, Axel Scherer, and William Krug

\begin{abstract}
Pairing high-quality factor $(Q)$ silicon-on-insulator microring resonators with rapidly tunable organic electrooptic claddings has allowed the first demonstration of a silicon-organic hybrid electrooptic reconfigurable optical add/drop multiplexer (ROADM). A coplanar electrode geometry provides up to 0.36 $\mathrm{GHz} / \mathrm{V}$ of electrooptic voltage tuning for each channel, corresponding to an electrooptic coefficient of $r_{33}=64 \mathrm{pm} / \mathrm{V}$ at wavelengths around $1550 \mathrm{~nm}$. Individual ring resonator devices have $40-\mu \mathrm{m}$ ring radii, $2.7-\mathrm{nm}$ free spectral range, and tuning ranges of $180 \mathrm{GHz}$. The $1 \times 4 \times 1$ ROADM has a footprint of less than $1 \mathrm{~mm}^{2}$ and has been shown to reconfigure in less than a microsecond.
\end{abstract}

Index Terms-Electrooptic devices, hybrid integrated circuit fabrication, integrated optics, nonlinear optics, optical polymers, resonator filters, wavelength-division multiplexing (WDM).

\section{INTRODUCTION}

$\mathbf{R}$ APIDLY tunable electrooptic (EO) Mach-Zehnder interferometers, resonant cavity microring resonator filters, and photonic bandgap crystal devices have been proposed as high speed optical interconnects for modulating and switching radio-frequency analog and digital optical signals in many commercial and military applications [1], [2]. Modulators made using inorganic EO materials such as silicon, gallium arsenide, and lithium niobate have recently demonstrated up to $50 \mathrm{GHz}$ modulation speeds; however, these devices continue to be performance limited due to intrinsically low EO activities, relatively slow (e.g., picoseconds) response times, and high velocity mismatch between electrical and optical waves [3], [4].

Ultrahigh bandwidth wavelength division multiplexing (WDM) for advanced computing systems can potentially be achieved using reconfigurable optical add/drop multiplexers

Manuscript received December 21, 2007; revised June 4, 2008. Current version published February 13, 2009.

J. Takayesu, P. Sullivan, J. Davies, and L. Dalton are with the University of Washington, Seattle, WA 98195 USA (e-mail: jocelynt@u.washington.edu).

M. Hochberg, T. Baehr-Jones, G. Wang, and A. Scherer are with the California Institute of Technology, Pasadena, CA 91125 USA.

E. Chan and W. Krug are with Boeing Phantom Works, Seattle, WA 98124 USA (e-mail: william.p.krug@boeing.com).

Y. Liao is with the University of Central Florida, Orlando, FL 32816 USA. Color versions of one or more of the figures in this paper are available online at http://ieeexplore.ieee.org

Digital Object Identifier 10.1109/JLT.2008.927776
(ROADMs). Existing ROADM technologies using microelectromechanical systems and thermo-optic devices, however, are limited to millisecond response times preventing their use in optical burst and packet switching or as reconfigurable optical circuits [5].

The very fast (tens of femtoseconds) optical response to electric stimuli intrinsic to organic EO materials makes this class of materials very attractive for high bandwidth operation [6]. These materials have demonstrated optical modulation up to $100 \mathrm{GHz}$ in commercial devices and up to $1.6 \mathrm{THz}$ experimentally [7], [8].

Organic EO materials for these applications have recently shown tenfold greater EO activity than their inorganic counterparts [9]. This trend of increasing EO activity with time continues through the theory-inspired design and nano-engineering of organic EO materials [10]. The high EO activity of organic materials has allowed for Mach-Zehnder interferometers with low halfwave voltages of less than $0.8 \mathrm{~V}$ to be realized; even lower halfwave voltages have recently been achieved [11], [12]. Other potential applications of organic EO materials include resonant devices such as ring resonators, etalons, and photonic bandgap periodic structures. Because the $\pi$-electrons of organic materials define both index of refraction and dielectric permittivity behavior, organic EO materials are a good velocity-matching medium between radio frequency signals and light; making these materials an attractive medium for EO switches and optical interconnects [13]. Moreover, EO materials are readily integrable with silicon-on-insulator (SOI) processing technologies and can be formulated to have optical transmission losses ranging from $0.2-2 \mathrm{~dB} / \mathrm{cm}$ at telecommunication wavelengths [14].

By integrating EO materials and waveguide-based optics in silicon, we aim to develop a new class of wafer-scale optical interconnects that will satisfy emerging high-speed communications and data processing needs. In this study, we demonstrate some of the advantages of integrating compact, high index contrast silicon optical waveguide technologies with highly active organic EO materials. An active EO hybrid ring resonator has been designed and fabricated as proof of concept. For broader applications, a multi-device, EO active, rapidly reconfigurable optical add/drop multiplexer with individually tunable microring resonators of good resonance quality and wide channel spacing has been designed and fabricated. In addition to having observed very high and controllable EO activities for voltage tuning of filter wavelengths, reasonable 
correlation between our results and published electrooptic values can be found among a variety of organic EO materials.

\section{DeVice Design AND PRedictions}

\section{A. Electro-Optic Materials}

When an electric field is applied to a noncentrosymmetrically poled EO polymer, polarization of the material is altered, resulting in a change of the refractive index of the material [14].

The relationship of index change $\Delta n$, to the applied field $E$, is dependent upon the electrooptic coefficient of the material $r_{33}$ and can be expressed as

$$
\Delta n=n_{\mathrm{o}}-n(E)=\frac{1}{2} r_{33} n_{\mathrm{o}}^{3} E
$$

where $n_{o}$ is the original index of refraction and $n(E)$ is the index of refraction as a function of the applied electric field [15]. Given a cladding with relatively modest activity of $100 \mathrm{pm} / \mathrm{V}$ and a refractive index of 1.73 , it is possible to estimate [using (1)] that the cladding will change in index by 0.0026 with the application of a $10-\mathrm{V} / \mu \mathrm{m}$ electric field.

Three EO active cladding systems are examined in this study. The first system is the chromophore compound YL156 doped 25\% in polymethylmethacrylate (PMMA); a system that has been shown to have moderately acceptable photostability (Fig. 1) [16]. The second system is the chromophore YL124 doped 25\% into Poly[Bisphenol A carbonate-co-4,4 '-(3,3,5-trimethylcyclohexylidene)diphenol carbonate] (APC); a system that has demonstrated moderate EO activity and thermal stability over $85{ }^{\circ} \mathrm{C}$ [17]. The third is YL124 doped $25 \%$ into a dendritic chromophore host, PSLD41; a system that has enhanced EO activity [18]. The EO activity of these materials have been measured using a reflection-based ellipsometry technique with a $1.3-\mu \mathrm{m}$ laser to have $r_{33}$ values of up to 50,120 , and $285 \mathrm{pm} / \mathrm{V}$, respectively [16], [19], [20]. Films of these types of organic materials have typically demonstrated low optical loss (less than $2 \mathrm{~dB} / \mathrm{cm}$ ) at telecommunication wavelengths and thermal stability over $70{ }^{\circ} \mathrm{C}$ [21].

\section{B. Optics Design}

Optical waveguides are used in a vast array of chip-scale photonic applications to transmit and manipulate light. To produce high quality silicon waveguides, we choose to use commercially available SOI wafers with a $1.4-\mu \mathrm{m}$-thick buried oxide layer and a 120-nm silicon top layer. The high refractive index contrast between the silicon core and buried oxide and polymer cladding layers allows compact waveguides, $0.5 \mu \mathrm{m}$ in width, to be realized [22]. With these waveguide dimensions, only one optical mode, polarized in-plane with the substrate surface, is supported in the 1.4-1.6- $\mu \mathrm{m}$ spectral range. Optical loss for these waveguides fall in the range of $4-7 \mathrm{~dB} / \mathrm{cm}$, depending on the cladding layer, however, the compact nature of these waveguides allow for the miniaturization of devices by at least tenfold compared, for instance, to polymer ring resonators, therefore lowering the impact of waveguide loss contribution to the overall loss of the device [23].

Illustrated in Fig. 2 is the modal profile of the silicon waveguide determined using a finite difference-based Hermitian

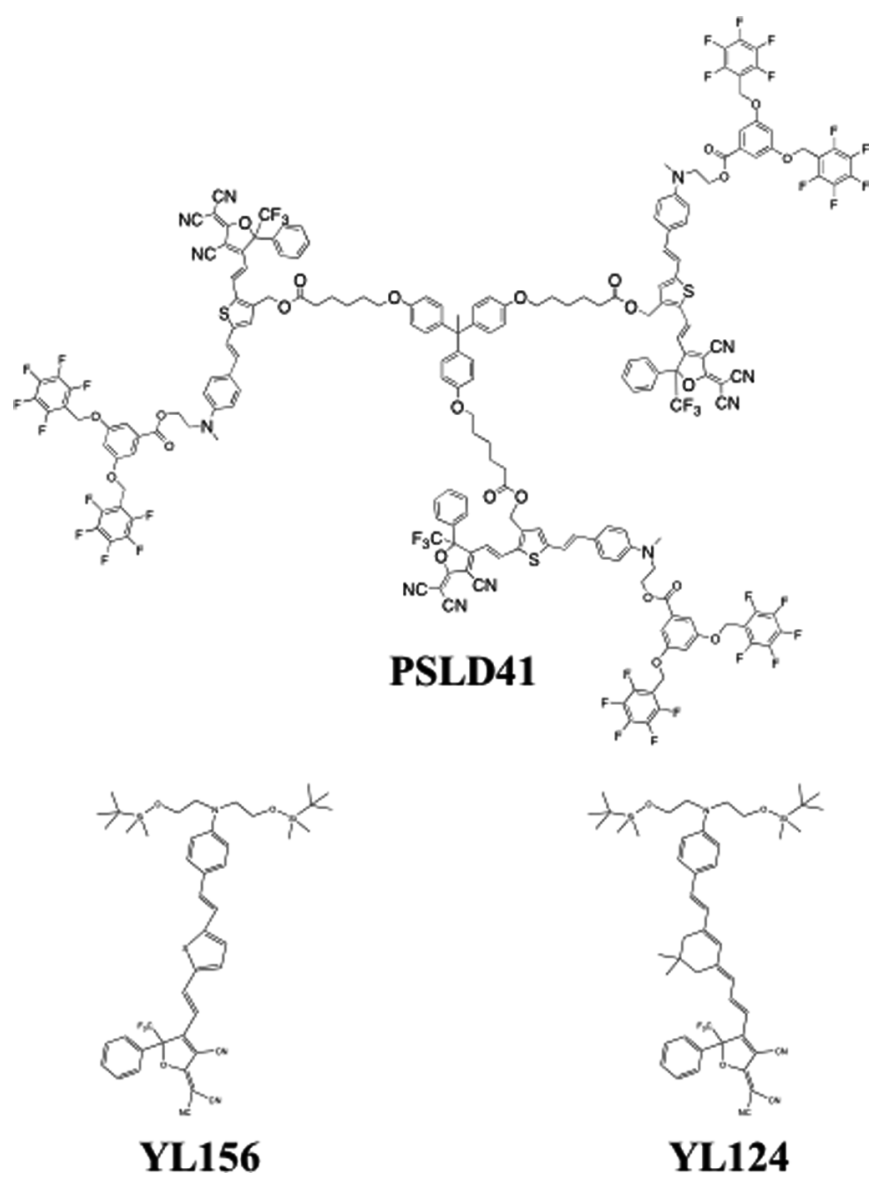

Fig. 1. Chemical structures of electrooptic molecules.

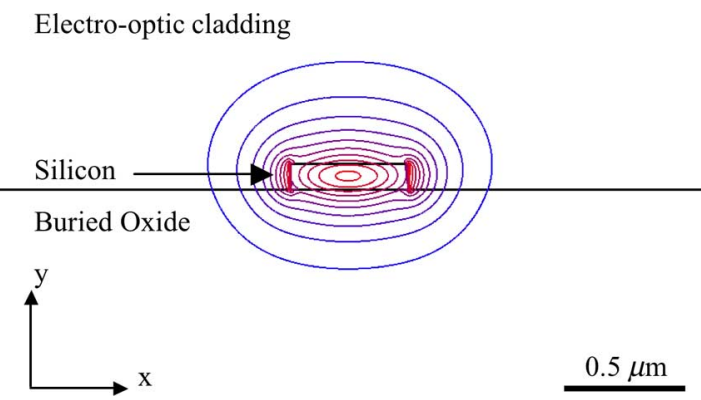

Fig. 2. Cross section of the modal profile of a silicon waveguide generated using finite element simulation. Contour lines of the modal field are drawn in $10 \%$ increments.

eigensolver [24]. The waveguide is designed to have more than $30 \%$ of the mode outside of the core so that perturbing the dielectric constant of the upper cladding induces a significant change in the effective index of the mode (Fig. 2). Using equations derived in an earlier publication, the effect of a small regional dielectric change of the upper polymer cladding on the effective index can be calculated [25].

The microring resonator is a resonant cavity device that traps wavelengths, $\lambda_{m}$, satisfying

$$
\lambda_{m}=\frac{L}{m} n_{\mathrm{eff}}(\lambda)
$$


where $m$ is an integer indicating the order of resonance, $L$ is the effective path length, and $n_{\text {eff }}(\lambda)$ is the wavelength-dependent effective index of the mode. Using (2), one can deduce that an increase in index causes resonant wavelengths to shift towards longer wavelengths and a decrease in index causes a shift towards shorter wavelengths.

When a ring resonator is placed within the evanescent coupling region of a waveguide, resonance wavelengths that satisfy (2) are coupled into and out of the ring resonator. Coupling efficiency can be lithographically controlled by varying the distance between the waveguide and ring. In these rings, a coupling distance of around $180 \mathrm{~nm}$ has been found to be the optimal distance for critical coupling.

Previous studies performed by our group to determine wavelength shift as a function of the change in cladding index have shown a reasonable correlation between theoretically predicted values and experimental results [25]. These studies used index matching fluids to extract a linear relationship between the cladding index change, $\Delta n_{\text {clad }}$, to the shift in wavelength position, $\Delta \lambda$, where

$$
\Delta \lambda=41.01(\mathrm{~nm}) \Delta n_{\text {clad }} 2 n_{\text {clad }} .
$$

This equation, in conjunction with (1), can be used to determine the EO activity factor, $r_{33}$, by monitoring $\Delta \lambda$ with applied voltage.

Silicon, which has a high index of refraction and low optical loss, has allowed for the fabrication of ring resonators with diameters less than $10 \mu \mathrm{m}$, FSRs greater than $15 \mathrm{~nm}$, and $Q$ values of over 1000000 [22], [26]. In these experiments, however, we intentionally quench $Q$ to values below 5000 to allow for increased reproducibility and reasonable switching speeds.

For experimental purposes, diffraction grating coupling was the chosen method for coupling a single TE mode into and out of the device waveguide [27]. Diffraction grating coupling enables simpler and more reproducible fabrication by eliminating the dicing and polishing steps needed for end-fire coupling. This method also reduces the footprint of a ring resonator device to less than $250 \mu \mathrm{m}^{2}$, allowing for remarkably dense device packing. Optical coupling of wavelengths around $1.55 \mu \mathrm{m}$ using this method was found to introduce losses of 5-10 dB, however, as noted in [27], loss from diffraction grating coupling can be greatly improved to less than $1 \mathrm{~dB}$ or $80 \%$ coupling efficiency. Combining holographic lens technology with diffraction grating coupling has shown to improve insertion loss to below $1.5 \mathrm{~dB}$ with best simulated results of $0.3 \mathrm{~dB}$ [28].

\section{Electrode Design and Theory}

For ring resonator-based modulators applied as single pole filters, a key figure of merit is the voltage required to shift the resonance by the full width at half maximum distance $\left(V_{\mathrm{FWHM}}\right)$. The intent is to maximize wavelength tuning sensitivity and tuning range while maintaining high modulation speeds. In these devices, electrodes coplanar to the waveguide are used to align and modulate the EO material surrounding the ring resonator waveguide. This geometry allows for a large electric field parallel to the TE mode to be applied across

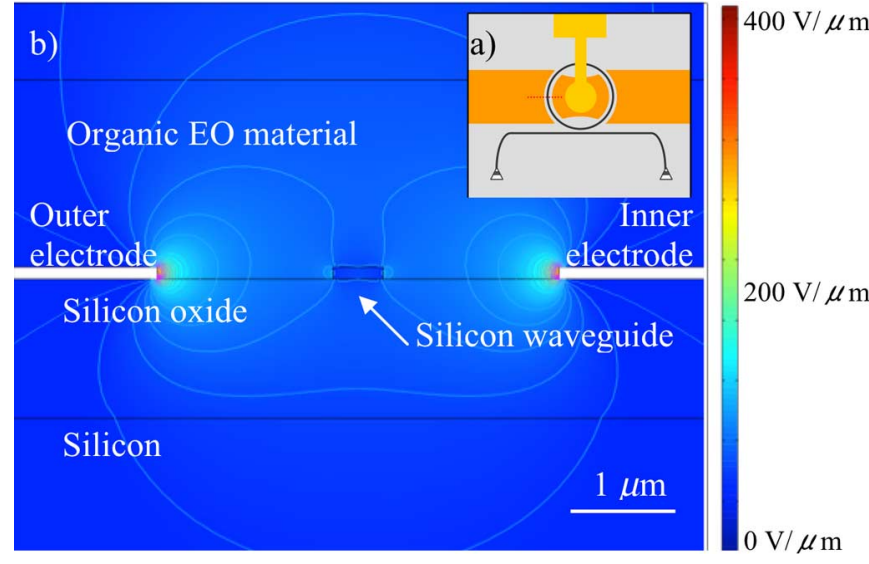

Fig. 3. (a) Inset: schematic top view of the electrode layout relative to the ring resonator waveguides. (b) Cross section of the device geometry, indicated by the red dotted line in (a) with superimposed contour plot of the electric field simulated using Comsol Multiphysics finite-element software).

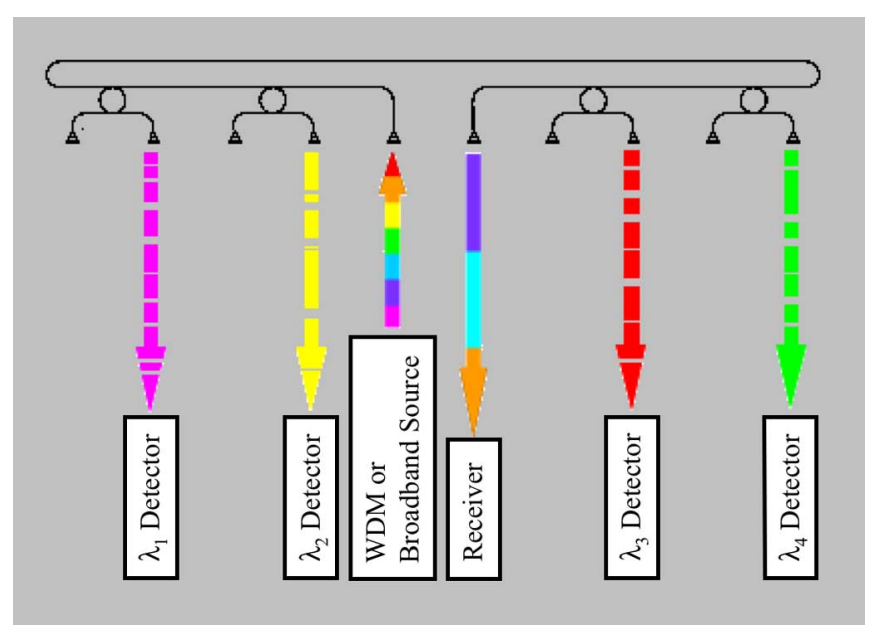

Fig. 4. Schematic of $1 \times 4 \times 1$ ROADM with individually tunable cascaded hybrid EO microring devices coupled to a common bus waveguide.

a short distance, thereby maximizing EO activity per volt [Fig. 3(a) and (b)]. Using finite-element method analysis, electrodes of $120-\mathrm{nm}$ height and $4-\mu \mathrm{m}$ spacing are found to produce an average field of $0.17 \mathrm{~V} / \mu \mathrm{m}$ per applied volt across the cladding material. To facilitate voltage application, the outer electrode is split into two electrodes and contacted using run outs and the inner electrode is accessed using a conductive via [Fig. 6(b)]. Modulation across the coupling regions is avoided to simplify the electrode design and minimize EO induced changes in coupling between the waveguide and ring [Fig. 6(a)].

\section{ROADM Design}

Of great interest to the data and telecommunications industries is to apply these devices as filters to rapidly and simultaneously add and drop various wavelength channels from a single multi-wavelength source coupled to a common (bus) waveguide in a chip-scale ROADM. To demonstrate this concept, we choose a simple cascaded microring device of four microring resonators coupled to a bus waveguide (Fig. 4). Broadband light is inserted into the bus and resonant 

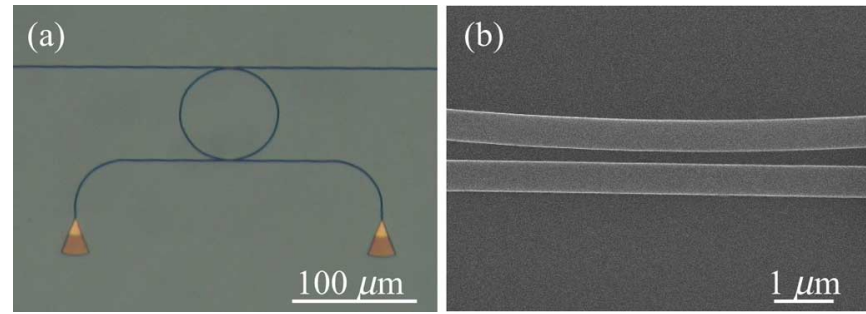

Fig. 5. (a) Top-view optical image of a silicon ring resonator, input/output waveguides, and diffraction grating couplers. (b) Scanning electron image of the coupling region with 180 -nm waveguide spacing.

wavelengths specific to each ring resonator are individually demultiplexed into separate drop waveguides. Resonance properties can be precisely tuned to select specific wavelengths by varying the radius of each ring or by tuning the cladding index. By reversing the input and output roles, this ROADM can also act as a multiplexer to combine various optical wavelength signals into a single bus waveguide.

When used as higher order pole filters with spectral efficiencies for ROADM applications, key figures of merit are the wavelength tuning sensitivity in $(\mathrm{GHz} / \mathrm{V})$, the tuning range in $\mathrm{GHz}$ relative to the free spectral range, FSR, of adjacent bands, and bandwidth, the speed of signal switching (bits/s). Colorless ROADMs also have a figure of merit based on finesse, $f$, the number of supported wavelength channels.

\section{FABRICATION}

The fabrication of single hybrid EO ring resonators and hybrid EO ROADMs can be summarized as a five-step process: 1) patterning of silicon waveguide core structures; 2 ) electrode formation; 3) EO material deposition; 4) electrode contact formation; and 5) EO material activation.

Silicon ring resonator devices $80 \mu \mathrm{m}$ in diameter with 500-nm waveguide widths are fabricated by means of electron beam patterning and reactive ion etching from electronic grade SOI wafers, purchased from Soitec, with top silicon thickness of approximately $120 \mathrm{~nm}$ on $1.4-\mu \mathrm{m}$ buried oxide [Fig. 6(b) ]; the details of this process can be found in one of our earlier publications [17]. The $1 \times 4 \times 1$ ROADM is patterned as four parallel cascaded ring resonator filters horizontally coupled to a common waveguide with 180-nm spacing [Figs. 4 and 5(b)].

The cross section of the hybrid ring resonator is shown in Fig. 6(b). Electrode material consisting of $10 \mathrm{~nm}$ of chromium followed by $110 \mathrm{~nm}$ of gold is deposited using electron beam evaporation followed by standard photolithography and wet etching to form the metal electrode pattern. EO material is prepared as a 25 weight $\%$ mixture of YL124 in host polymer Poly[Bisphenol A carbonate-co-4,4 '-(3,3,5-trimethylcyclohexylidene)diphenol carbonate] dissolved with cyclopentanone to form a 12 solids weight $\%$ solution. After complete dissolution overnight, the solution is filtered through a $0.2-\mu \mathrm{m}$ PTFE filter. Solutions of 25\% YL156 in PMMA and 25\% YL124 in PSLD41 are prepared in an identical manner. Films of these materials around $1.2-\mu \mathrm{m}$ thick are spun-cast onto the SOI devices substrates, baked for one minute at $110^{\circ} \mathrm{C}$ and vacuum cured overnight at $85^{\circ} \mathrm{C}$. To increase cladding layer thickness
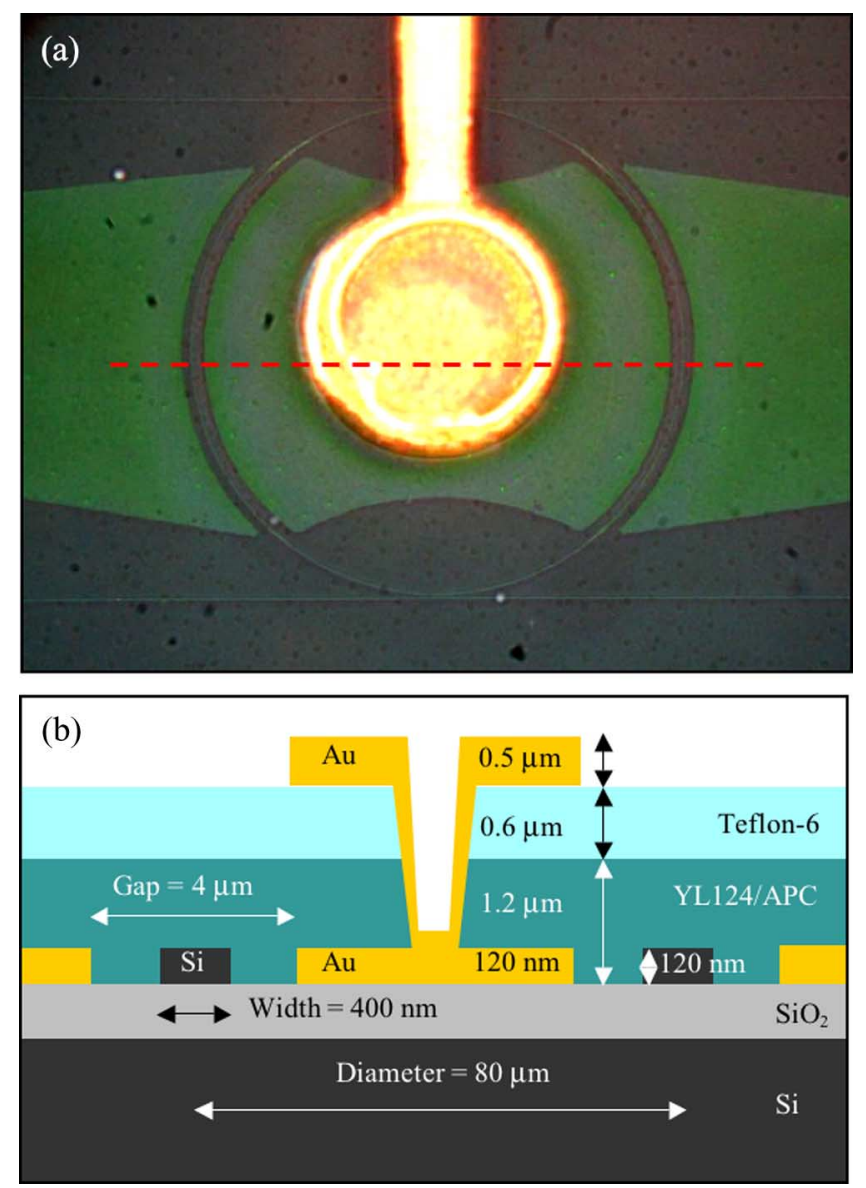

Fig. 6. (a) Top-view optical image of fabricated EO microring resonator device. (b) Cross-section schematic, indicated by the dotted red line in (a) of a single hybrid microring resonator device.

and protect the EO layer from further processing steps, a 0.6- $\mu \mathrm{m}$ Teflon AF 601S2-1-6 film is spun-cast over the EO layer and cured. A 100-nm-thick gold hard mask is sputtered on top of this film and vias are patterned using photolithography. To access the inner electrode, vias through the polymer layers are etched using a reactive ion plasma of oxygen and helium gas. To contact the inner electrode, a 400-nm layer of gold is sputtered that plates the via sidewalls. Electrode runouts on the surface are patterned by photolithography followed by wet chemical etching. A second $0.6-\mu \mathrm{m}$ Teflon layer is applied to the top of the device to protect the upper electrodes from delaminating and contact areas are exposed using $\mathrm{O}_{2} / \mathrm{He}$ plasma reactive ion etching.

The device illustrated in Fig. 6(a) and (b) is poled and tuned by application of a voltage across the EO polymer cladding between the inner and outer electrodes [Fig. 3(b)]. Leakage currents due to impurities in the organic materials and dielectric breakdown further contribute to the complexity of calculating the applied field and may contribute to less than estimated tuning efficiencies measured for device operation.

During contact poling of these devices, the temperature of the sample is first raised to just below its glass transition temperature $\left(\mathrm{T}_{\mathrm{g}} \approx 146^{\circ} \mathrm{C}\right.$ for YL124/APC) in order to provide rotational mobility of the chromophore. A direct current $(\mathrm{dc})$ voltage is then applied to create a $30-60-\mathrm{V} / \mu \mathrm{m}$ field between 


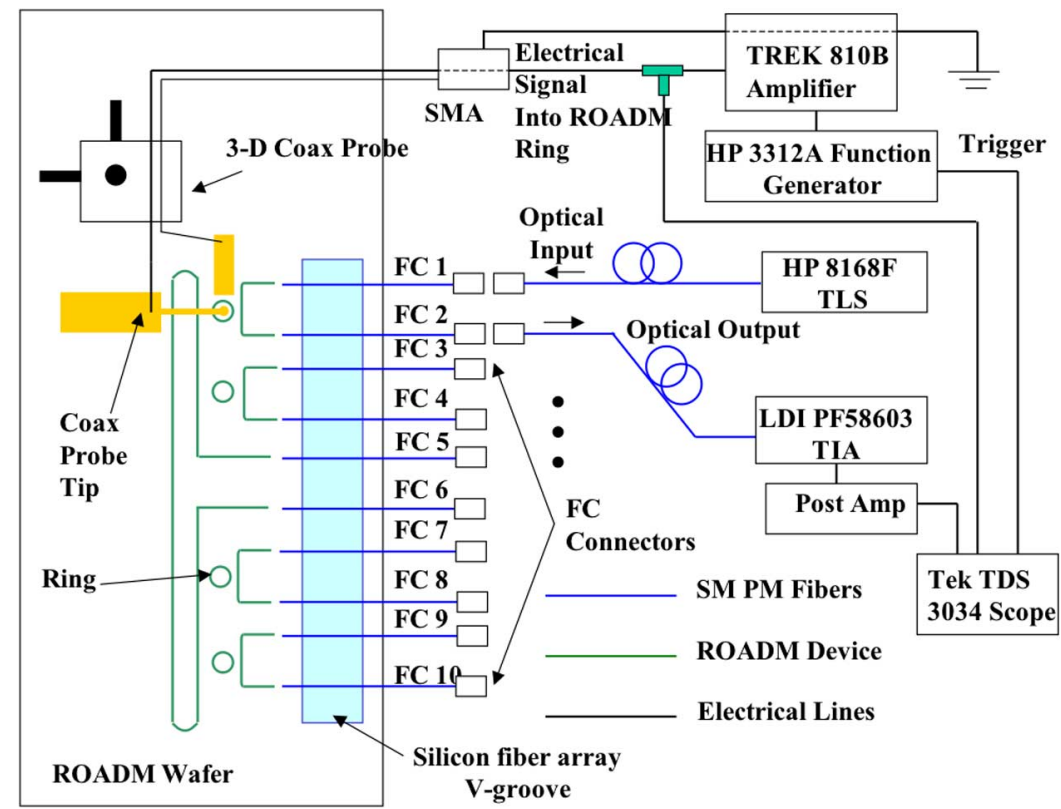

Fig. 7. Test setup schematic for ROADM device testing.

the inner and outer electrodes and the sample is held at this temperature under the electric field for two minutes after which the sample is cooled back down to room temperature and the field is removed.

\section{Device Performance}

\section{A. Hybrid Silicon-Organic Microring Resonator}

To prepare for testing, samples are mounted onto an optical stage system and coupled out-of-plane to a single-mode optical fiber array using grating couplers (Fig. 7). For spectral characterization, the light source used is an ILX Lightwave amplified spontaneous emission (ASE) broadband source centered at $1550 \mathrm{~nm}$ with $20-\mathrm{mW}$ output optical power (not pictured). The output signal is analyzed using an ANDO AQ6316 Optical Spectrum Analyzer with 0.05-nm resolution. In Fig. 8(a), the spectral scans of each of the four microring resonator devices, made using YL124/APC EO material, are overlayed and can be compared to the spectral scan of the bus waveguide for the $1 \times 4 \times 1$ ROADM in Fig. 8(b).

The direct correlation of the resonance peaks of each filter with resonance peaks of the ROADM shows that the spectrum of the ROADM is a linear combination of its constituent device spectra.

The total optical loss for a diffraction grating coupled EO-clad silicon microring resonator and the coupled $1 \times 4 \times 1$ ROADM was measured to be approximately -10 and $-20 \mathrm{~dB}$, respectively. As shown in Fig. 8(a), the free spectral range for single EO clad microring resonator devices is $2.74 \mathrm{~nm}$, the $3-\mathrm{dB}$ bandwidth for these devices is approximately $0.51 \mathrm{~nm}$, and the resonance peaks display up to a $17.5-\mathrm{dB}$ drop in transmission power. These details imply $Q$ values for these devices around 3000 and finesse values above 5 .

Variations in electrode alignment and spacing may cause effective poling fields to vary from one device configuration to the next. As a result, subtle differences in index may be significant

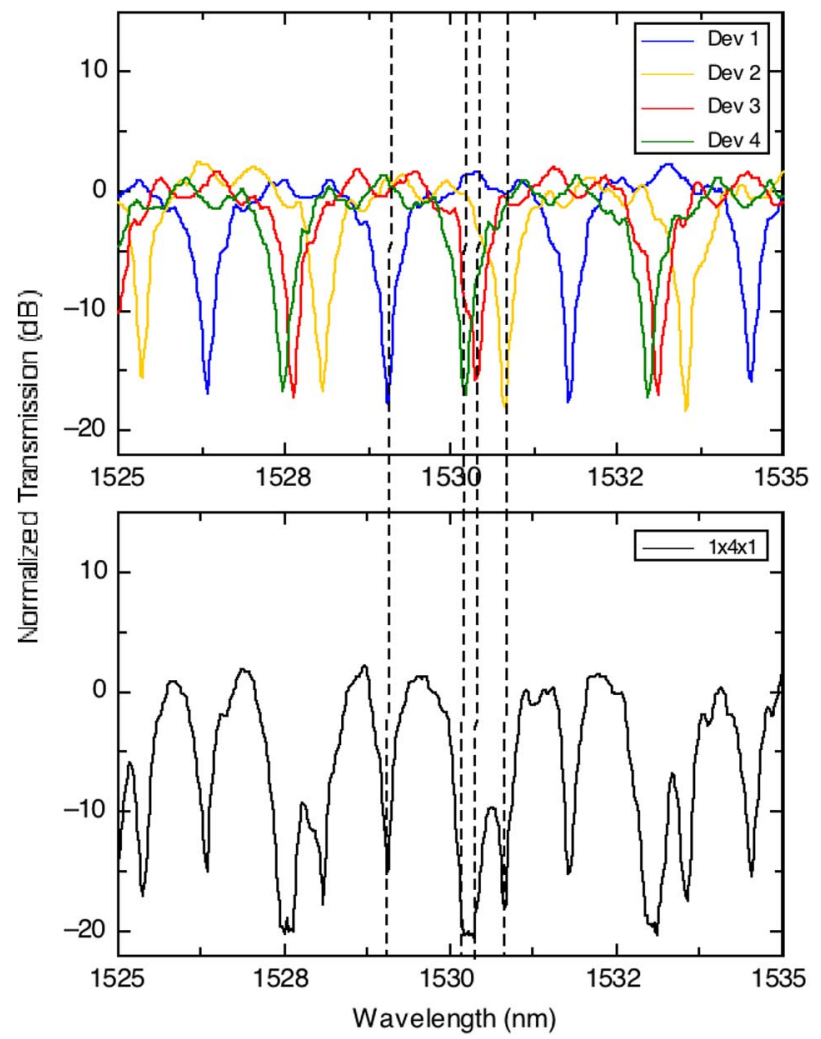

Fig. 8. (a) Overlayed resonance spectra of devices $1,2,3$, and 4 coated with YL124/APC EO material. (b) Resonance spectrum of the $1 \times 4 \times 1$ ROADM device.

enough to bestow upon each device a distinct resonance spectrum as is observed among devices in Fig. 8(a). In this experiment, devices 1-4 are poled simultaneously; however, in future applications, one can individually tune filters electrically while monitoring optical output to attain the desired filtering pattern of a ROADM device. 


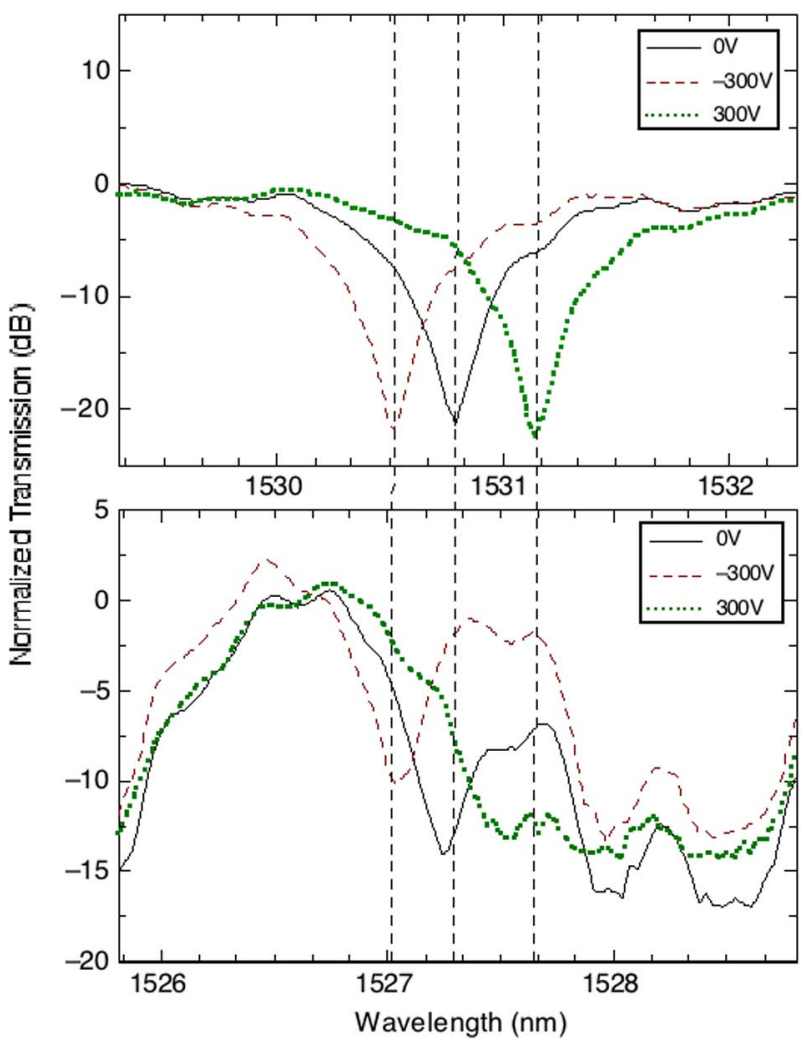

Fig. 9. (a) Overlayed resonance spectra of device 4 under various applied fields. (b) Overlayed resonance spectra of the $1 \times 4 \times 1$ bus waveguide device with modulation of device 4.

To demonstrate EO tuning $(\mathrm{GHz} / \mathrm{V})$, a dc voltage is applied to a device with YL124/APC cladding using a TREK 810B power supply/amplifier. By coupling an optical signal to the input and output ports of device 4 , the dc modulation of device 4 is monitored showing a clear shift to the left when- $300 \mathrm{~V}$ is applied, a return to the original position when voltage is removed, and a clear shift to the right when $300 \mathrm{~V}$ is applied [Fig. 9(a)]. This $0.67 \mathrm{~nm}$ shift with $600-\mathrm{V}$ dc modulation at $1.55 \mu \mathrm{m}$ is equivalent to $0.14-\mathrm{GHz} / \mathrm{V}$ tuning. The $r_{33}$ value of the $\mathrm{EO}$ material is determined to be $33 \mathrm{pm} / \mathrm{V}$ using (1) and (3). Devices 1, 2, and 3 yield similar EO tuning ranging from 0.131 to $0.160 \mathrm{GHz} / \mathrm{V}$.

To verify that resonance tuning is a result of an electrooptic effect, the position of a set of four selected resonance peaks is monitored after application of $0,-50,+50,-100$, $+100,-200$, and $+200 \mathrm{~V}$ in succession. The average shift in wavelength position of the four resonance peaks with each applied voltage for a device coated with the highly active YL124/PSLD41 material is presented in Fig. 10.

Voltage tuning of this device shows a linear dependence of wavelength position with applied voltage in either polarity indicating that the EO tuning of this device is primarily dependant on the second-order nonlinear effect (Fig. 10). This supports studies that have found the contribution from the third-order nonlinear effect to not be significant in EO devices made using similar materials [29]. When a voltage opposite to the polarity of the poling field is applied, tuning of $0.56 \mathrm{GHz} / \mathrm{V}$ is observed towards shorter wavelengths, indicating a decrease in index using (2). When a voltage of the same polarity as the poling field is applied, tuning of $0.14 \mathrm{GHz} / \mathrm{V}$ is observed towards longer wavelengths. This discrepancy in tuning efficiency may be due to

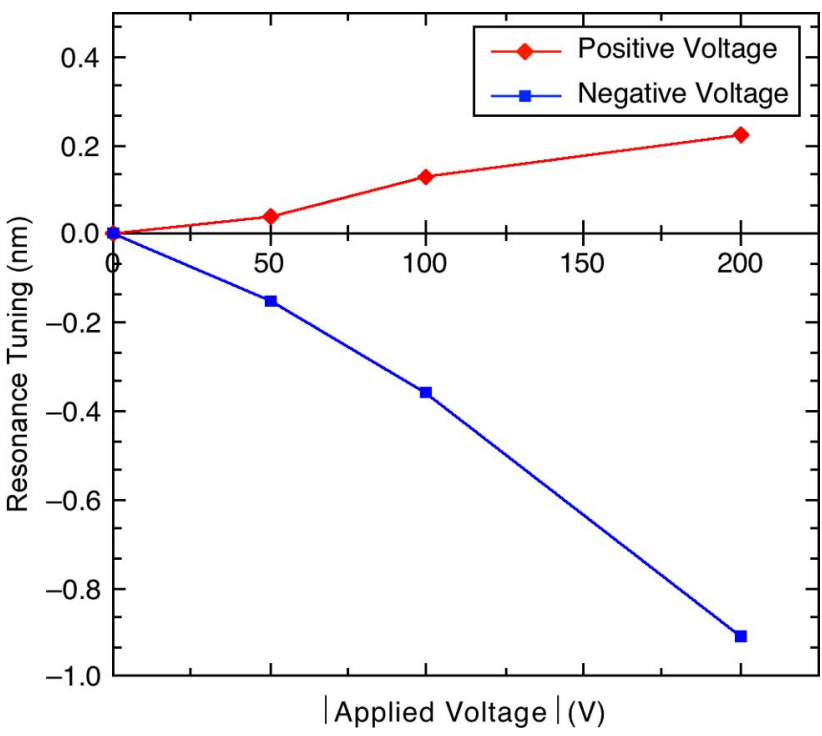

Fig. 10. Voltage tuning of resonant wavelengths around $1550 \mathrm{~nm}$. Illustrated in red (diamonds) is tuning using voltage of the same polarity to that applied during poling and illustrated in blue (squares) is tuning using voltage of opposite polarity to that applied during poling.

TABLE I

EXPECTED AND MEASURED ElECTROOPTIC ACTIVITY FOR VARIOUS DEVICES AT $1550 \mathrm{~nm}$

\begin{tabular}{lccccc}
\hline Material & $\begin{array}{c}\text { Poling } \\
\text { Field } \\
(\mathrm{V} / \mu \mathrm{m})\end{array}$ & $\begin{array}{c}\text { Expected } \\
\mathrm{r}_{33} \\
(\mathrm{pm} / \mathrm{V})\end{array}$ & $\begin{array}{c}\text { Measured } \\
\mathrm{r}_{33} \\
(\mathrm{pm} / \mathrm{V})\end{array}$ & $\begin{array}{c}\text { Tuning } \\
(\mathrm{GHz} / \mathrm{V})\end{array}$ & $\begin{array}{c}Q \\
\text { value }\end{array}$ \\
\hline $\begin{array}{l}\text { YL156/ } \\
\text { PMMA }\end{array}$ & 49 & 19 & $13 \pm 2$ & 0.06 & 1890 \\
$\begin{array}{l}\text { YL124/ } \\
\text { APC }\end{array}$ & 45 & 44 & $32 \pm 1$ & 0.17 & 3000 \\
$\begin{array}{l}\text { YL124/ } \\
\text { PSLD41 }\end{array}$ & 37 & 85 & $55 \pm 6$ & 0.36 & 1750 \\
\hline
\end{tabular}

greater conductivity of the material in the direction of poling and is more frequently observed when using EO materials with high chromophore content. Overall tuning values were measured by applying the positive and negative voltages of a set value and determining the difference in resonance peak position.

EO ROADMs fabricated using the system of YL124 chromophore doped into the dendritic chromophore PSLD41 display EO tuning activities up to $0.36 \mathrm{GHz} / \mathrm{V}$ with measured $r_{33}$ values ranging from 53 to $64 \mathrm{pm} / \mathrm{V}$ over six devices tested. This twofold increase of EO activity compared to the YL124/APC system $\left(r_{33}=32 \mathrm{pm} / \mathrm{V}\right)$ exactly corresponds to the relative strengths of measured literature values given the magnitude of applied field and spectral wavelength (Table I). The relatively photo-stable system, YL156/PMMA displays $40 \%$ of the activity of YL124/APC, a value only slightly lower than the $43 \%$ prediction from literature values. In all three material systems, the 30\% lower than expected EO performance observed is most likely due to the avoidance of poling over the coupling regions of the device, which accounts for roughly $30 \%$ of the resonator cladding area.

At high modulation voltages ( $\pm 300 \mathrm{~V}$ DC), these devices exhibit an extended tuning range of $1.44 \mathrm{~nm}$ or $180 \mathrm{GHz}$ at 1.55 $\mu \mathrm{m}$ wavelengths. Fields above $70 \mathrm{~V} \mu \mathrm{m}$ are not applied due to 
limitations set by the silicon breakdown threshold voltage of the waveguide core.

When a large electric field (greater than $50 \mathrm{~V} \mu \mathrm{m}$ ) is applied to the hybrid EO device, the resonance spectrum, in some cases, exhibits a small but significant degree of drift. The magnitude of drift is found to be significantly lowered by reducing the strength of the modulating electric field, operating under an inert atmosphere, and by using EO materials with superior optical stability. Details of these studies will be reported in a later publication.

Wafer-level alternating current (ac) modulation for ROADM devices is performed using the test setup illustrated as a block diagram in Fig. 7. The key objective of this wafer level testing setup is to verify the ac modulation characteristics of the ROADM devices using triangular waveforms with amplitude up to $300 \mathrm{~V}$ and higher. The other objective is to provide a high dynamic range detection capability for testing high loss devices. The LDI PF58603 is an integrated PINFET receiver that provides the system with detection sensitivity better than $-60 \mathrm{dBm}$ in the $1550-\mathrm{nm}$ wavelength range. A standard post amplifier is used to increase the output voltage into the Tektronix TDS 3034 scope. A coaxial probe is used to make contact to the inner and outer electrodes of the ring resonator. The coaxial probe is connected to a TREK $810 \mathrm{~B}$ operating in the amplifier mode and an HP 3312A function generator is used to drive the TREK $810 \mathrm{~B}$ triangular waveform at different frequencies. The output triangular waveform from the TREK $810 \mathrm{~B}$ is adjustable to over $300 \mathrm{~V}$. An array of ten single-mode $\mathrm{PM}$ fibers on a silicon V-groove is aligned to the ROADM device wafer mounted on a high precision alignment stage. After the fiber alignment, measurement begins by adjusting the HP8168 tunable laser (TLS) to a wavelength near a resonance peak of the ring resonator, then a triangular waveform from the TREK $810 \mathrm{~B}$ is turned on and the amplitude is slowly adjusted until a modulated waveform is observed on the Tektronix TDS 3034 multichannel scope. Using a coaxial T-connector, the driver triangular waveform and the ring resonator's response waveform are captured simultaneously on the TDS 3034 scope. The ac modulation of an EO hybrid ring resonator with YL124/PSLD41 cladding is captured in Fig. 11(a). The upper trace is the triangular wave form of the modulating voltage source and the lower trace is the optical response of the ring resonator. The response trace of the ring resonator shows a secondary middle peak between two major peaks for each triangular pulse period; this characteristic verifies the ring resonator has achieved ac modulation.

To determine response time, another figure of merit for EO modulators, a square wave ac voltage is applied across the device [Fig. 11(b)]. The rise and fall times are measured to 55 and $67 \mathrm{~ns}$, respectively. AC tuning is performed up to $3 \mathrm{MHz}$; however, this limitation is due to the bandwidth of the TREK 810B high voltage amplifier and the LDI PF5860 high-sensitivity PINFET.

\section{B. $1 \times 4 \times 1 R O A D M$}

To demonstrate the functionality of the $1 \times 4 \times 1$ ROADM, ring resonator filters must be capable of being independently modulated with low crosstalk. By coupling to the bus device while modulating only device 4 , it is observed that only one
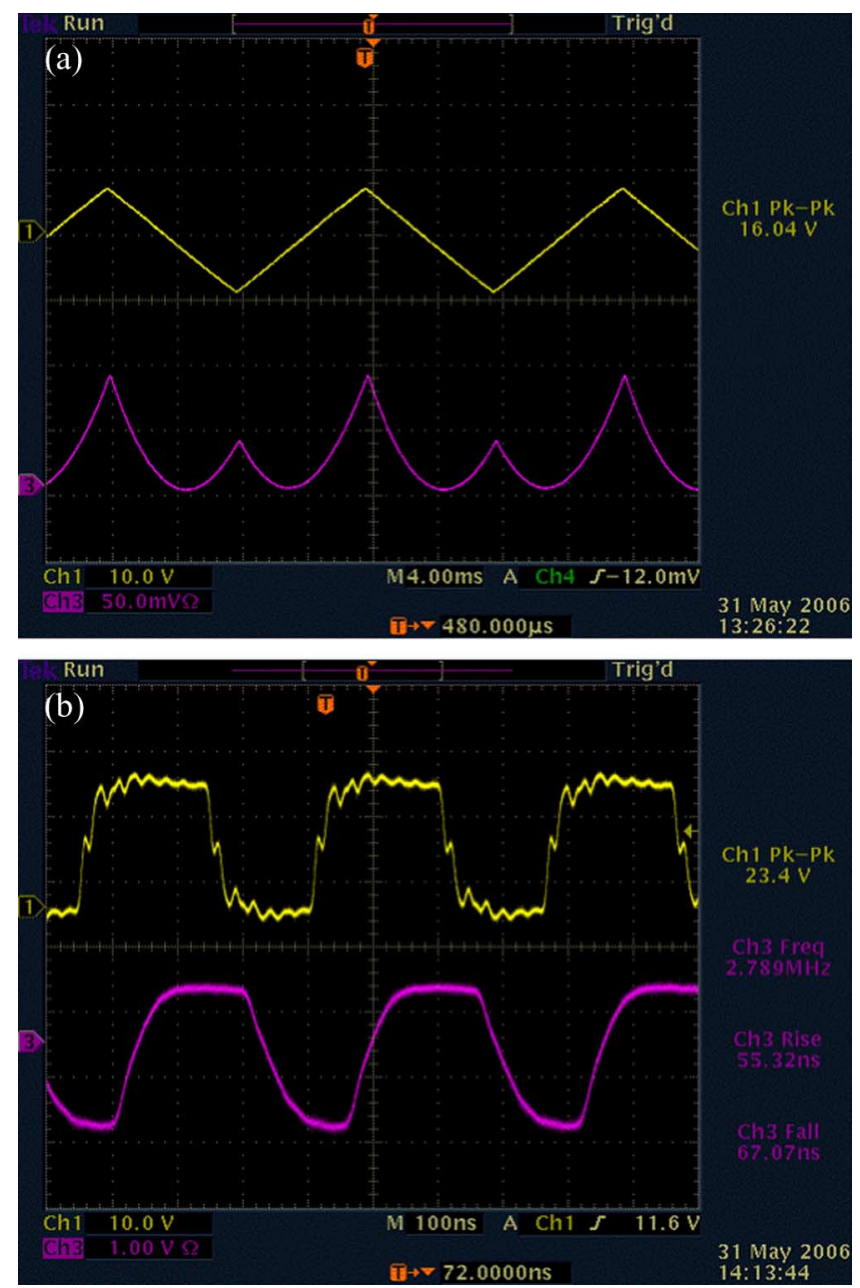

Fig. 11. (a) Waveform of ac voltage applied to the device (top) and modulated optical output intensity measured from the drop waveguide at a fixed wavelength around $1550 \mathrm{~nm}$. (b) Higher frequency ac voltage applied as a square wave bit pattern (top) and time response of optical output signal measured from the drop waveguide at a fixed wavelength around $1550 \mathrm{~nm}$.

peak modulates significantly while the other peaks maintain the same position [Fig. 9(b)]. Although the resonance of device 4 overlaps with another device spectrum, there is a definite match in shifting behavior between Fig. 9(a) and (b) and general match in peak position. In this study, all four ring resonator filters can be modulated or wavelength tuned independent of one another demonstrating the complete functionality of our $1 \times 4 \times 1$ ROADM device. Our measured tuning shows that these devices support tuning of four $50 \mathrm{GHz}$ channels with at least $2.8 \mathrm{MHz}$ bandwidth and $0.36 \mathrm{GHz} / \mathrm{V}$ tuning in addition to functionality as a 1st order passband filter.

When kept at room temperature under vacuum for over three months, this EO ROADM maintains over $80 \%$ of its original activity indicating stability over time.

\section{CONCLUSION}

The authors have developed and demonstrated a first of its kind hybrid silicon-organic electrooptic ROADM device, with strong EO tuning characteristics and sub-microsecond response times. For use as low-drive voltage modulators, modest improvements, including the use of materials with higher EO 
activity, closer electrode spacing, and ring resonators with larger $Q$ values, for example, would result in dramatically reduced $V_{\text {FWHM }}(3 \mathrm{~dB})$ drive voltages. Recently, organic EO materials have demonstrated over $300 \mathrm{pm} / \mathrm{V}$ EO activity and polymer-clad SOI microring devices with $Q$ values of $57 \mathrm{k}$ have been developed; therefore, lower $V_{\mathrm{FWHM}}$ targets can certainly be achieved in the near future [9], [22]. Electrode spacing can be reduced using standard procedures in high-resolution photomask and alignment technology or by using e-beam patterning. Even more effective means of reducing electrode gaps employs the use of doped silicon electrodes to confine and modulate a 100-nm EO waveguide core [17].

To further improve ROADM tuning characteristics, only a several-fold enhancement would be required to tune over a 50-GHz DWDM channel spacing. This enhancement can readily be achieved with Vernier tuning approaches and/or reduced electrode gaps. The use of smaller diameter SOI rings to extend the FSR to support 40 channels at $50-\mathrm{GHz} / \mathrm{channel}$ and multi-pole SOI microring filters to optimize the spectral efficiency will lead to rapidly EO-tunable, DWDM-compatible hybrid EO on SOI device systems.

The integration of nonlinear organic materials with existing silicon photonic technologies has thus enabled the development of hybrid silicon-organic microring resonator devices that may well satisfy the need for greater speed, lower drive power, and smaller sized voltage-controlled filter devices to be used in tomorrow's demanding telecommunication, data communication, and aerospace applications.

\section{ACKNOWLEDGMENT}

The authors would like to gratefully acknowledge D. Koshinz, B. Capron, H. Hager, J. Nielsen, R. Lawson, and J. Luo for their helpful discussion and support.

\section{REFERENCES}

[1] C. Vazquez, S. Vargas, J. Manuel, S. Pena, and P. Corredera, "Tunable optical filters using compound ring resonators for DWDM," IEEE Photon. Technol. Lett., vol. 15, no. 8, pp. 1085-1087, Aug. 2003.

[2] A. F. J. Levi, "Optical interconnects in systems," Proc. IEEE, vol. 88, no. 6, pp. 750-757, Jun. 2000.

[3] Q. Xu, S. Manipatriuni, B. Schmidt, J. Shakya, and M. Lipson, "12.5 Gbit/s carrier-injection-based silicon micro-ring silicon modulators," Opt. Exp., vol. 15, pp. 430-436, Jan. 2007.

[4] Y. Shi, "Micromachined wideband lithium-niobate electrooptic modulators," IEEE Trans. Microw. Theory Tech., vol. 54, no. 2, pp. 810-815, Feb. 2006.

[5] E. Klein, D. Geuzebroek, H. Kelderman, G. Sengo, N. Baker, and A. Dreissen, "Reconfigurable optical add-drop multiplexer using microring resonators," IEEE Photon. Technol. Lett., vol. 11, no. 11, pp. 2358-2360, Nov. 2005.

[6] L. R. Dalton, "Organic electrooptic materials," Pure Appl. Chem., vol. 76, no. 7-8, pp. 1421-1433, 2004.

[7] M. R. Fetterman, J. A. Grata, R. Dinu, M. Koenig, A. D. Visnansky, and W. L. Kiser, Jr., "Electro-optic polymer modulators as passive $\mathrm{mm}$ wave detectors," Proc. SPIE, vol. 6472, pp. 1-13, 2007.

[8] M. Lee, H. E. Katz, C. Erben, D. M. Hill, P. Gopalan, J. D. Heber, and D. J. McGee, "Broadband modulation of light using an electo-optic polymer," Science, vol. 298, pp. 1401-1403, Nov. 2002.

[9] T.-D. Kim, J.-W. Kang, J. Luo, S.-H. Jang, J.-W. Ka, N. Tucker, J. B. Benedict, L. R. Dalton, T. Gray, R. M. Overney, D. H. Park, W. N. Herman, and A. K.-Y. Jen, "Ultralarge and thermally stable electro-optic activities from supramolecular self-assembled molecular glasses," J. Amer. Chem. Soc., vol. 129, pp. 488-489, Jan. 2007.

[10] L. R. Dalton, A. K. Jen, P. A. Sullivan, B. E. Eichinger, B. H. Robinson, and A. M. Chen, "Theoretically-inspired rational design of electrooptic materials," Nonlinear Opt. Quantum Opt., vol. 35, pp. 1-19, 2006.
[11] C. Zhang, L. R. Dalton, M. Oh, H. Zhang, and W. H. Steier, "Low V electrooptic modulators from CLD-1: Chromophore design and synthesis, material processing, and characterization," Chem. Mater., vol. 13, pp. 3043-3050, 2001.

[12] L. Dalton, Nov. 2007, Revisiting Organic Optical Nonlinearity Leads to a New Class of Materials SPIE Newsroom [Online]. Available: http:// spie.org/x 17559.xml

[13] K. Okamoto, Fundamentals of Optical Waveguides. San Diego, CA: Academic, 2000.

[14] L. Dalton, "Nonlinear optical polymeric materials: From chromophore design to commercial applications ," Adv. Polym. Sci., vol. 158, pp. $1-86,158,2002$.

[15] B. E. A. Saleh and M. C. Teich, Fundamentals of Photonics. New York: Wiley, 1991.

[16] Y. Liao, C. Anderson, P. Sullivan, A. Akelaitis, B. Robinson, and L. Dalton, "Electro-optical properties of polymers containing alternating nonlinear optical chromophores and bulky spacers," Chem. Mater., vol. 18, pp. 1062-1067, 2006.

[17] T. Baehr-Jones, M. Hochberg, G. Wang, R. Lawson, Y. Liao, P. A Sullivan, L. Dalton, A. K.-Y. Jen, and A. Scherer, "Optical modulation and detection in slotted silicon waveguides," Opt. Express, vol. 13, no. 14, pp. 5216-5226, Jun. 2005.

[18] P. A. Sullivan, H. Rommel, Y. Liao, B. C. Olbricht, A. J. P. Akelaitis, K. A. Firestone, J.-W. Kang, J. Luo, J. A. Davies, D. H. Choi, B. E. Eichinger, P. J. Reid, A. Chen, A. K.-Y. Jen, B. H. Robinson, and L. R. Dalton, "Theory-guided design and synthesis of multichromophore dendrimers: An analysis of the electro-optic effect," J. Amer. Chem. Soc., vol. 129, pp. 7523-7530, May 2007.

[19] P. A. Sullivan, B. C. Olbright, A. Akelaitis, A. Mistry, Y. Liao, and L. Dalton, "Tri-component Diels-Alder polymerized dendrimer glass exhibiting large, thermally stable, electro-optic activity," J. Mater. Chem. vol. 17, pp. 2899-2903, Apr. 2007.

[20] L. R. Dalton, P. A. Sullivan, D. H. Bale, and B. C. Olbricht, "Theory-inspired nano-engineering of photonic and electronic materials: Noncentrosymmetric charge-transfer electro-optic materials," Solid-State Electron., vol. 51, pp. 1263-1277, Aug. 2007.

[21] L. Dalton, "Integrated optics/electronics using electro-optic polymers," Mater. Res. Soc. Symp. Proc., vol. 817, pp. 181-193, 2004.

[22] T. Baehr-Jones, M. Hochberg, C. Walker, and A. Scherer, "High-Q ring resonators in thin silicon-on-insulator," Appl. Phys. Lett., vol. 85, no. 16, pp. 3346-3347, Oct. 2004.

[23] P. Rabiei, W. Steier, C. Zhang, and L. Dalton, "Polymer micro-ring filters and modulators," J. Lightw. Technol., vol. 20, no. 11, pp. 1968-1975, Nov. 2002

[24] A. Taflove, Computational Electrodynamics. Boston, MA: Artech House, 1995.

[25] T. Baehr-Jones, M. Hochberg, C. Walker, E. Chan, D. Koshinz, W. Krug, and A. Scherer, "Analysis of the tuning sensitivity of silicon-oninsulator optical ring resonators," J. Lightw. Technol., vol. 23, no. 12 pp. 4215-4221, Dec. 2005

[26] M. Borselli, T. Johnson, and O. Painter, "Beyond the Rayleigh scattering limit in high-Q silicon microdisks: Theory and experiment," Opt. Exp., vol. 13, no. 5, pp. 1515-1530, Mar. 2005.

[27] G. Roelkens, D. Van Thourhout, and R. Baets, "High efficiency grating coupler between silicon on insulator waveguides and perfectly vertical optical fibers," Opt. Exp., vol. 32, no. 11, pp. 1495-1497, Jun. 2007.

[28] C. Gunn, "CMOS photonics for high-speed interconnects," IEEE Micro., vol. 26, no. 2, pp. 58-66, Mar/Apr. 2006.

[29] N. P. Bhatambrekar, L. Dalton, J. Luo, A. K.-Y. Jen, and A. Chen, "Third-order nonlinearity contribution to electro-optic activity in polymer materials in a constant bias field," Appl. Phys. Lett, vol. 88, pp. 1-3, Aug. 2005.

Jocelyn Takayesu received the B.S. degree in chemistry and mathematics from the University of Puget Sound, Tacoma, WA, in 2003 and the M.S. and Ph.D. degrees in materials chemistry and nanotechnology from the University of Washington, Seattle, in 2006 and 2008, respectively.

Her current research interests include synthesis and characterization of electrooptic materials and fabrication of nanophotonic electrooptic devices.

Michael Hochberg received the B.S. degree in physics and the M.S. and Ph.D degrees in applied physics from the California Institute of Technology (Caltech), Pasadena, in 2002, 2005, and 2006, respectively. 
As an undergraduate, he cofounded Luxtera, a venture-funded company that has raised over $\$ 50 \mathrm{~m}$ to commercialize silicon photonics, and he spent a year there full-time after finishing his undergraduate work. As a graduate student, he has worked on developing ultrafast nonlinear optical devices using silicon photonics. This research agenda has included the development of all-optical switches operating at terahertz bandwidth, the integrated optical rectification based detectors, and a variety of other linear and nonlinear optical devices. $\mathrm{He}$ has recently joined the faculty at the University of Washington in electrical engineering.

Dr. Hochberg was the recipient of a National Science Foundation (NSF) Graduate Research Fellowship and a Caltech Upper Class Merit Award. He was awarded the Demetriades-Tsafka Prize in Nanotechnology for the best thesis by a graduating Ph.D. student in the field of Nanotechnology.

Tom Baehr-Jones received the B.S. degree in physics and the M.S. and Ph.D. degrees in applied physics from the California Institute of Technology, Pasadena, in 2002, 2005, and 2006, respectively.

He cofounded Luxtera, Inc., in 2001, a firm that specializes in the design of integrated optics components. There, he helped design such components with the assistance of FDTD software. He also worked in the Scherer Lab as a Postdoctoral Scholar from 2006 to 2007. Currently, he is a Research Scientist with Prof. M. Hochberg at the University of Washington. His research interests include integrated optics and novel geometries for the use of nonlinear optical polymers.

Eric Chan received the B.S. degree (with honors) in electrical engineering and computer science from the University of California, Berkeley, in 1973, and the M.S. and Ph.D. degrees in electrical engineering from Columbia University, New York, in 1975 and 1979, respectively.

From 1979 to 1987, he was a Senior Member of Technical Staff with the Optoelectronic Group, AT\&T Engineering Research Center, Princeton, NJ. He joined Boeing in 1987. Currently, he is a Boeing Technical Fellow working with the Optical Technology Group, Boeing Phantom Works, Seattle, WA. His research interests include design, manufacturing, packaging, and characterization of optoelectronic devices and modules for aerospace applications. He holds 16 U.S. patents, has authored/coauthored over 60 technical/conference papers, and wrote a book chapter in the Handbook of Compound Semiconductors (Noyes, 1995).

Guangxi Wang received the B.S. degree in electrical and computer engineering from Lafayette College, Easton, PA, in 2003 and the M.S degree in electrical engineering from the California Institute of Technology, Pasadena, in 2004, where he is currently working toward the Ph.D. degree in electrical engineering.

His research interests include integrated nonlinear optics and related applications on silicon-polymer hybrid platform.

Philip Sullivan received the B.S. degree in chemistry from Montana State University, Bozeman, in 2001 and the Ph.D. degree in organic chemistry and nanotechnology from the University of Washington, Seattle, in 2005.

Currently, he is a Post-Doctoral Research Associate with the University of Washington. His research focus is in the area of organic materials for photonic applications including the design, synthesis and characterization of materials (polymers/dendrimers/small molecules) for electrooptic and all optical information processing. His research interests include biophotonics, photovoltaics, and organic synthesis.
Yi Liao received the B.S. degree from Nankai University, China, and the Ph.D. degree from the University of Massachusetts, Amherst, in 2001.

He was a Postdoctoral Fellow with Dr. J. Miller's group at the University of Utah and then Dr. L. Dalton's group at the University of Washington. He is currently an Assistant Professor with the Chemistry Department, University of Central Florida, Orlando. His research interests include organic and hybrid electronic, optic, and magnetic materials.

Josh Davies received the B.S. degree in chemistry from Simon Fraser University, Burnaby, BC, Canada, in 2003. He is currently working toward the Ph.D. degree in chemistry at the University of Washington, Seattle.

His current research interests include design, synthesis, and characterization of electrooptic materials.

Larry Dalton received the B.S. and M.S. degrees (with highest honors) from the Honors College of Michigan State University, Lansing, in 1965 and 1966, respectively, and the A.M. and Ph.D. degrees from Harvard University, Cambridge, MA, in 1971, all in chemistry.

He is currently the George B. Kauffman Professor of Chemistry and Electrical Engineering with the University of Washington, Seattle, where he also directs the National Science Foundation (NSF) Science and Technology Center on Materials and Devices for Information Technology Research. He is the author of over 500 scientific publications.

Dr. Dalton is a Fellow of the American Association for the Advancement of Science and the Optical Society of America. He was the recipient of the IEEE/LEOS William Streifer Award, the American Chemical Society Award in the Chemistry of Materials, the American Chemical Society Richard C. Tolman Medal, and a Michigan State University Distinguished Alumni Award. Current Federal Advisory Service includes the Defense Science Board Advisory Group on Electron Devices, the NSF Mathematical and Physical Sciences Directorate Advisory Committee, the Nanotechnology Technical Advisory Group of the President's Council of Advisors on Science and Technology, and the Committee of Visitors of the Division of Materials Research of the NSF

Axel Scherer, photograph and biography not available at the time of publication.

William Krug received the Ph.D. degree in applied physics and organic chemistry from the Johns Hopkins University, Baltimore, MD.

He joined the Boeing High Tech Center in 1986 to lead research in nonlinear optical switching. He is an Associate Technical Fellow for Optical Technology in Information, Electronics, and Avionics with Boeing Phantom Works, Seattle, WA. He was a Principal Investigator for a Chip-Scale Wavelength Division Multiplexing (WDM) DARPA contract with the California Institute of Technology and the University of Washington to demonstrate chip scale system-on-a-chip wavelength routing for networks on military platforms. He leads Boeing's efforts to standardize emerging WDM-LANs for avionics across all DOD platforms and is also actively pursuing approaches for optics to provide information assurance. He holds several U.S. patents and has coauthored over 40 papers and patent applications.

Dr. Krug is a member of SPIE and the American Chemical Society. He was a recipient of a Boeing Special Invention Award. 